\title{
Correction to: Measuring Efficiency and Productivity Change in the Turkish Electricity Distribution Sector
}

\author{
Yetkin Cinar and Tekiner Kaya
}

Correction to:

Chapter "Measuring Efficiency and Productivity Change in the Turkish Electricity Distribution Sector" in:

A. B. Dorsman et al. (eds.), Applied Operations Research

and Financial Modelling in Energy, https://doi.org/10.1007/978-3-030-84981-8_5

In the original version of the chapter, table 1 was inadvertently split into two tables (table 1 and table 2). This has now been rectified and the subsequent tables have been renumbered. 